\title{
Could IFN- $\gamma$ predict the development of residual pleural thickening in tuberculous pleurisy?
}

\author{
I. Gerogianni, M. Papala, P. Tsopa, P. Zigoulis, A. Dimoulis, \\ K. Kostikas, T. Kiropoulos, K.I. Gourgoulianis
}

\begin{abstract}
Could IFN- $\gamma$ predict the development of residual pleural thickening in tuberculous pleurisy? I. Gerogianni, M. Papala, P. Tsopa, P. Zigoulis, A. Dimoulis, K. Kostikas, T. Kiropoulos, KI Gourgoulianis.

Background. The aim of our study was to identify predictive factors for the development of residual pleural thickening (RPT) in patients with tuberculous pleurisy (TP).

Methods. A retrospective study of patients with pleural tuberculosis. The clinical and radiological characteristics, and measurements of microbiological and biochemical parameters or markers such as adenosine deaminase (ADA), interferon- $\gamma($ IFN- $\gamma)$ and vascular endothelial growth factor (VEGF) in pleural fluid were studied.
\end{abstract}

Results. Thirty one patients ( 24 male and 7 female) with a mean age of 55.9 years were studied. There were $25(80.6 \%)$ patients with RPT $>2 \mathrm{~mm}$ and $6(19.4 \%)$ patients without RPT. Ten patients $(32.2 \%)$ had RPT $\geq 10$ $\mathrm{mm}$. The rate of pleural thickening was less in small effusions $(p<0.05)$. IFN $-\gamma$ was higher in patients with RPT $\geq 10 \mathrm{~mm}(\mathrm{p}<0.05)$ in comparison with those with RPT $<10 \mathrm{~mm}$.

Conclusions. Pleural fluid IFN- $\gamma$ may deserve further investigation in order to build up preventive and therapeutic strategies against RPT and its clinical complications.

Monaldi Arch Chest Dis 2008; 69: 1, 18-23.

Keywords: Tuberculosis pleurisy, Pleural thickening, Adenosine deaminase, Interferon- $\%$.

Department of Respiratory Medicine, Medical School, University of Larissa, Larissa, Greece.

Correspondence: Irini Gerogianni MD, PhD, Respiratory Medicine Department, University of Thessaly, Greece, Mezourlo, 41110 Larissa, Greece; e-mail: igerogianni@yahoo.gr

\section{Introduction}

The incidence of pleural tuberculosis is difficult to determine with precision. In the United Kingdom, pleural involvement is seen in less than $10 \%$ of cases of infection with Mycobacterium tuberculosis [1]. Pleural disease is seen more frequently in ethnic minorities with active tuberculosis living in the western world. However, the epidemiology of pleural tuberculosis may be different in other countries, particularly in Africa, due to the impact of HIV infection [2]. Tuberculosis treatment is based on the administration of a combination of antituberculosis medications. Although antituberculosis therapeutic regimens have successful results, the treatment of pleural tuberculosis (PT) is followed by radiographic residual pleural thickening (RPT) in about half of the patients [3]. Corticosteroids and therapeutic thoracentesis (TT) have been added to pharmacological antituberculosis treatment in an attempt to reduce RPT, but contradictory results have been reported.

In PT, pleural fluid concentrations of biological parameters such as adenosine deaminase (ADA), interferon- $\gamma(\mathrm{IFN}-\gamma)$ and vascular endothelial growth factor (VEGF) are increased as a result of the inflammation seen in tuberculosis. ADA is an enzyme related to purine catabolism and higher levels are seen in lymphocytes. There is a positive relationship between ADA levels and pleural fluid CD4+ cells as well as lymphocyte differentiation [4]. Likewise, it is thought that IFN- $\gamma$ is produced by the CD4+ lymphocytes from patients with tuberculous pleuritis [5]. Finally, a few reports have shown an increase of VEGF in patients with tuberculosis. VEGF, a homodimeric, heparin-binding glycoprotein, is one of the major mediators of angiogenesis and vascular permeability [6]. It seems that different biological parameters participate in the development of pleural inflammation and fibrosis secondary to pleural tuberculosis.

The aim of this retrospective study was to investigate the relationship between the concentrations of ADA, IFN- $\gamma$ and VEGF, along with the clinical, biochemical, and microbiological characteristics of pleural fluid in the development of RPT.

\section{Methods}

A total of 31 patients were diagnosed with tuberculous pleural effusions in the Respiratory Department of University Hospital of Larissa between 2003 and 2005. Inclusion criteria were completion of antituberculosis treatment and absence 
of other radiologic pleural abnormalities. Demographic, clinical, and radiologic data was obtained from the medical records. TST results were also collected.

The diagnosis of tuberculous effusions was based on the presence of positive stain or culture of Mycobacterium tuberculosis (TB) in the pleural fluid, sputum, or pleural biopsy specimen, or in the presence of typical caseating granulomas on a pleural biopsy specimen. When a pleural biopsy was not performed, we considered patients to have tuberculous pleurisy if they met the following criteria: (1) adenosine deaminase levels in pleural fluid of $>40 \mathrm{U} / \mathrm{L}$, (2) the exclusion of any other cause of pleural effusion; and (3) the response to antituberculous treatment.

In pleural fluid samples obtained from the first thoracentecis performed in each patient, pleural fluid biochemistry (lactate dehydrogenase [LDH], glucose, proteins, $\mathrm{pH}$ ), cell count, and microbiology (culture in Lowenstein-Jensen medium of pleural fluid and pleural biopsy) were studied. Moreover, aliquots of pleural fluid were immediately centrifuged at $1500 \mathrm{~g}$ for $15 \mathrm{~min}$ at $4^{\circ} \mathrm{C}$ and the supernatants were stored at $-80^{\circ} \mathrm{C}$ for $\mathrm{ADA}$, IFN- $\gamma$ and VEGF measurements. Pleural fluid ADA measurements were performed with the colorimetric method of Giusti [7]. IFN- $\gamma$ and VEGF levels were measured with a commercially available enzyme-immunosorbent assay kit (Biosource Europe S.A.) according to the manufacturer's protocol. The lower limits of detection for IFN- $\gamma$ and VEGF were $0.03 \mathrm{IU} / \mathrm{mL}$ and $<5 \mathrm{pg} / \mathrm{mL}$, respectively.

The radiologic size of the pleural effusion was defined as small when the fluid contour was seen above the costophrenic angle, large when the effusion occupied at least half of the hemithorax, and medium when the level of fluid was between the two. All patients received isoniazid, rifampin, pyrazinamide, and ethambutol for the first two months which was followed by isoniazid and rifampin for the next 4 months. No patient was treated by thoracentesis for relief.

RPT was measured in the lower lateral hemithorax of a posteroanterior chest radiograph at the level of an imaginary line intersecting the diaphragmatic dome, in the first month after the treatment was concluded. Two criteria for pleural thickness were used to classify patients by the presence or absence of RPT. First, RPT was defined as a pleural thickness of $>2 \mathrm{~mm}$, a value considered radiologically abnormal. Analyses were repeated using RPT defined as a pleural thickness of $\geq 10 \mathrm{~mm}$ because this may have significant impact in pulmonary function [8]. Measurements were made by two independent observers and agreement between the two was evaluated.

\section{Statistical analysis}

Data is presented as mean \pm standard deviation (SD), unless otherwise stated. Parameters were compared with Student's $t$-tests for normally distributed data with Mann-Whitney U tests for skewed data. Qualitative variables were compared with the chisquare method using Yates' correction and the Fischer Exact Test was used as needed. Measurements of RPT on chest radiographs were made by two independent observers and agreement between the two was evaluated using the kappa index.

\section{Results}

31 patients with PT were included in the study: 24 were male and 7 were female with a mean age of 55.9 years (range 16-84 years). There were 25 $(80.6 \%)$ patients with RPT $>2 \mathrm{~mm}$ and $6(19.4 \%)$ patients without RPT. Ten patients $(32.2 \%)$ had $\mathrm{RPT} \geq 10 \mathrm{~mm}$.

Table 1 shows the comparisons of clinical and microbiological characteristics, PPD values and smoking habits between the group with RPT $>2$ $\mathrm{mm}$ and the group without RPT. Patients with residual pleural thickening were older than those without RPT but there was no statistical difference. There was also no statistical difference in gender, clinical and microbiological findings, smoking rates and PPD values between the two groups.

Table 2 shows the comparisons of radiological findings in patients with RPT $>2 \mathrm{~mm}$ or without RPT. The rate of pleural thickening was lower in small effusions $(p<0.05)$. Effusions were accompanied by parenchymal infiltrations on posteroanterior roentgenograms in 15 patients. There was no difference in the RPT incidence between those with parenchymal infiltrations and those without parenchymal infiltrations on posteroanterior roentgenograms. Tables 3 and 4 present pleural

Table 1. - Comparison of clinical, microbiologic characteristics and smoking rates in patients with residual pleural thickening (RPT) $>2 \mathrm{~mm}$ and those without pleural thickening

\begin{tabular}{lcc}
\hline & $\begin{array}{c}\text { with RPT } \\
\mathbf{n}(\%)\end{array}$ & $\begin{array}{c}\text { without RPT } \\
\mathbf{n}(\%)\end{array}$ \\
\hline age, ys \pm & $59.3 \pm 17.8$ & $44.3 \pm 27.6$ \\
male & $19 / 25(76.00)$ & $5 / 6(83.3)$ \\
history of post TB & $4 / 25(16.00)$ & $1 / 6(16.6)$ \\
pleuritic chest pain & $14 / 25(56.00)$ & $1 / 6(16.6)$ \\
cough & $19 / 25(76.00)$ & $5 / 6(83.3)$ \\
fever & $15 / 25(60.00)$ & $3 / 6(50)$ \\
fatigue & $8 / 25(32.00)$ & $3 / 6(50)$ \\
weight loss & $12 / 25(48.00)$ & $2 / 6(33.3)$ \\
sputum & $12 / 25(48.00)$ & $3 / 6(50)$ \\
night sweats & $13 / 25(52.00)$ & $2 / 6(33.3)$ \\
dyspnea & $18 / 25(72.00)$ & $2 / 6(33.3)$ \\
TST positive & $15 / 25(60.00)$ & $4 / 6(66.6)$ \\
Non-smoker & $7 / 25(28.00)$ & $3(50)$ \\
smoker & $18 / 25(72.00)$ & $3(50)$ \\
tobacco consumption (p/ys) & $45.4 \pm 27.1$ & $25.7 \pm 12.9$ \\
positive Lw PF & 10 & 1 \\
\hline
\end{tabular}

TST $=$ tuberculin skin test. Lw = culture in LŏwensteinJensen medium. $\mathrm{PF}=$ pleural fluid. 
Table 2. - Comparison of radiological findings in patients with residual pleural thickening (RPT) $>2 \mathrm{~mm}$ and those without residual pleural thickening

$\begin{array}{ccc}\text { with RPT } & \text { without RPT } & p \text { value } \\ \text { n }(\%) & \text { n }(\%) & \end{array}$

\begin{tabular}{|c|c|c|c|}
\hline \multicolumn{4}{|l|}{ initial size of $P E$} \\
\hline small & $5 / 25(20.00)$ & $4 / 6(66.6)$ & $<0.05$ \\
\hline medium & $14 / 25(56.00)$ & $2 / 6(33.4)$ & NS \\
\hline massive & $6 / 25(24.00)$ & 0 & \\
\hline \multicolumn{4}{|l|}{ localisation of $P E$} \\
\hline right & $15 / 25(60.00)$ & $3 / 6(50)$ & NS \\
\hline left & $10 / 25(40.00)$ & $3 / 6(50)$ & NS \\
\hline accompanied by parenchymal infiltrations & $13 / 25(52.00)$ & $2 / 6(33.3)$ & NS \\
\hline
\end{tabular}

$\mathrm{PE}=$ pleural effusion

fluid biochemical values, cell counts, levels of biomarkers and lymphocyte rates in patients with and without RPT. There were no statistical differences between these two groups.

Moreover, patients were divided in those with $\mathrm{RPT} \geq 10 \mathrm{~mm}$ and those with RPT $<10 \mathrm{~mm}$. The main clinical and radiological findings are shown in table 5. The pleural fluid biochemical values, cell count, and biological parameters are shown in table 6 . IFN- $\gamma$ was the only marker that was higher in patients with RPT $\geq 10 \mathrm{~mm}(p<0.05)$ in comparison with those with RPT $<10 \mathrm{~mm}$ (figure 1 ). ROC curve analysis showed that no single value of baseline IFN- $\gamma$ levels represents an optimal cut-off

Table 3. - Comparison of pleural fluid characteristics in patients with and without RPT $>2 \mathrm{~mm}$

\begin{tabular}{lcc}
\hline & $\begin{array}{c}\text { with RPT } \\
\text { N = 25 }\end{array}$ & $\begin{array}{c}\text { without RPT } \\
\mathbf{N}=\mathbf{6}\end{array}$ \\
\hline LDH (U/L) & $530 \pm 334.6$ & $467 \pm 301$ \\
Glucose (mg/dl) & $87.00 \pm 25.1$ & $81.8 \pm 15.2$ \\
Total Pr (g/dl) & $4.7 \pm 0.87$ & $5.34 \pm 0.46$ \\
pH & $7.3 \pm 0.07$ & $7.33 \pm 0.08$ \\
CELLS (*103) & $4.1 \pm 2.8$ & $5.2 \pm 3.1$ \\
ADA (UI/L) & $65.6 \pm 27.00$ & $85.1 \pm 15.4$ \\
INF- $\boldsymbol{\gamma}(\mathbf{U I} / \mathbf{m l})$ & $28.4 \pm 28.2$ & $26 \pm 24.4$ \\
VEGF (pgr/ml) & $624 \pm 278.8$ & $586 \pm 383$ \\
\hline
\end{tabular}

Values given as mean $\pm \mathrm{SD}$. point for the prediction of effusions that will present $\mathrm{RPT} \geq 10 \mathrm{~mm}$ (AUC 0.624, 95\% CI 0.433$0.790)$. However, IFN- $\gamma$ values $>63.6 \mathrm{IU} / \mathrm{ml}$ present $90.5 \%$ specificity, yet with $20.0 \%$ sensitivity.

\section{Discussion}

Our results showed that IFN- $\gamma$ values were higher in the group with RPT of $\geq 10 \mathrm{~mm}$ compared to the group with RPT $<10 \mathrm{~mm}$, whereas no other of the baseline characteristics of the pleural fluid at the time of the diagnosis was related to RPT. Moreover, our results showed that, from the 31 patients studied with pleural tuberculosis, 25 had developed residual pleural tuberculosis when their therapy was completed.

The incidence of RPT after antituberculosis treatment varies between studies. Lee et al. reported RPT in $10 \%$ of the cases, whereas Barbas et al. reported 14\% and Soler et al. reported 72\% [9-11]. Wyser et al. reported $60 \%$ and Pablo et al reported $42,8 \%[12,13]$. Recently, Uskul et al. showed that the rate of development of pleural thickening was $52 \%$ [14]. This variation can be attributed to the lack of a uniform concept of residual pleural thickening: some defined a pleural thickening of $\geq 2 \mathrm{~mm}$ as abnormal, and others require a thickness $\geq 10 \mathrm{~mm}$.

In our study, we found that neither age nor sex influenced the later development of RPT. Male and older subjects predominated among patients with RPT in our study, but the result was not statistically significant. Soler et al. reported that patients with RPT > $2 \mathrm{~mm}$ were mainly male subjects

Table 4. - Lymphocyte rates in the cytological examinations of pleural fluid samples

\begin{tabular}{lccc}
\hline Total lymphocyte rates in pleural fluid & with RPT $\mathbf{n}(\boldsymbol{\%})$ & without RPT $\mathbf{n}(\boldsymbol{\%})$ & total $\mathbf{n}(\boldsymbol{\%})$ \\
\hline$<82 \%$ & $11 / 25(44.00)$ & $3 / 6(50)$ & $11(42)$ \\
$>82 \%$ & $14 / 25(56.00)$ & $3 / 6(50)$ & $15(57.7)$ \\
total (n) & 25 & 6 & 26 \\
\hline
\end{tabular}


Table 5. - Comparison of main clinical, radiological and microbiological characteristics in patients with $\mathrm{RPT} \geq 10 \mathrm{~mm}$ and those with RPT $<10 \mathrm{~mm}$

\begin{tabular}{|c|c|c|}
\hline & $\begin{array}{c}\mathrm{RPT} \geq 10 \mathrm{~mm} \\
n=10\end{array}$ & $\begin{array}{c}\text { RPT }<10 \mathrm{~mm} \\
n=21\end{array}$ \\
\hline age, yr & $63.4 \pm 15.7$ & $53.00 \pm 21.8$ \\
\hline male & 8 & 16 \\
\hline \multicolumn{3}{|l|}{ radiologic size } \\
\hline small & 1 & 8 \\
\hline moderate & 6 & 13 \\
\hline large & 3 & 0 \\
\hline positive PPD & 5 & 13 \\
\hline positive Lw PF & 3 & 7 \\
\hline
\end{tabular}

$\mathrm{Lw}=$ culture in Lŏwenstein-Jensen medium. $\mathrm{PF}=$ pleural fluid. PPD = tubeculin skin test.

Table 6. - Comparison of pleural fluid characteristics in patients with RPT $\geq 10 \mathrm{~mm}$ and those with RPT $<10 \mathrm{~mm}$

\begin{tabular}{lccc}
\hline & $\begin{array}{c}\text { RPT } \\
\boldsymbol{n}=\mathbf{1 0}\end{array}$ & $\begin{array}{c}\text { RPT }<\mathbf{~ 1 0 ~} \mathbf{~ m m} \\
\boldsymbol{n}=\mathbf{2 1}\end{array}$ & $\boldsymbol{p}$ value \\
\hline LDH (U/L) & $540.2 \pm 433.8$ & $507.3 \pm 270.2$ & $\mathrm{NS}$ \\
Glucose (mg/dl) & $87.1 \pm 29.5$ & $85.6 \pm 20.7$ & NS \\
Total Pr (g/d) & $4.7 \pm 0.9$ & $4.8 \pm 0.83$ & NS \\
pH & $7.28 \pm 0.063$ & $7.35 \pm 0.083$ & NS \\
CELLS & $4.6^{*} 10^{3} \pm 3.2 * 10^{3}$ & $3.7 * 10^{3} \pm 2.1 * 10^{3}$ & NS \\
ADA (UI/L) & $64.5 \pm 22.4$ & $71.7 \pm 27.8$ & NS \\
INF- $\gamma(\mathbf{U I} / \mathbf{m l})$ & $36.9 \pm 28.3$ & $23.7 \pm 26.4$ & $<0.05$ \\
VEGF $(\mathbf{p g r} / \mathbf{m l})$ & $573.00 \pm 223$ & $638.3 \pm 326.4$ & NS \\
\hline
\end{tabular}

Values given as mean \pm SD.

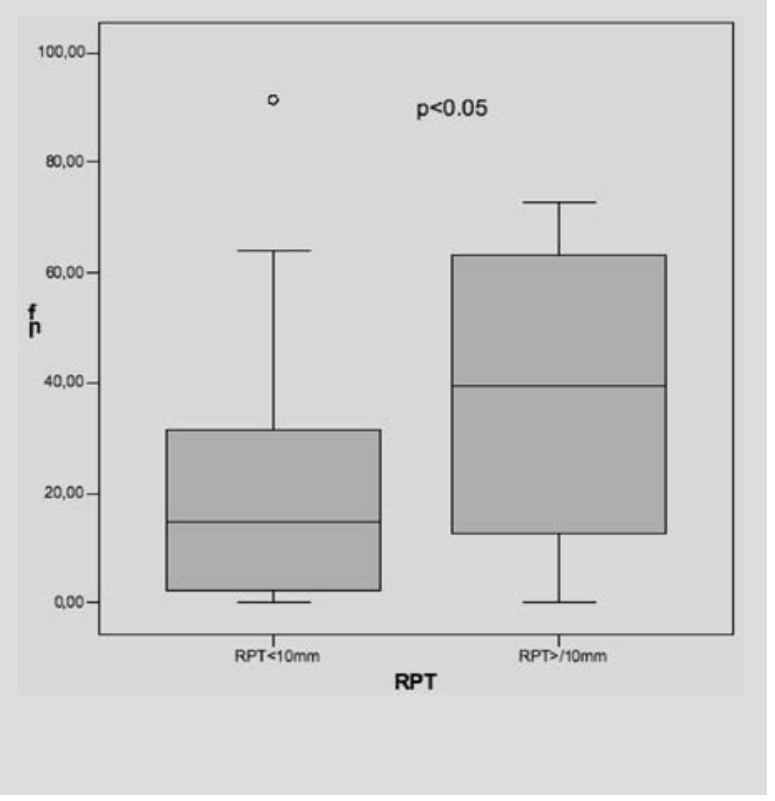

Fig. 1. - IFN- $\gamma$ concentration in pleural fluid of patients with and without $\mathrm{RPT} \geq 10 \mathrm{~mm}$. whereas Pablo et al. obtained significant sex difference for RPT > $10 \mathrm{~mm}$ [10, 12]. Interestingly, our results concur with the radiological findings of Uskul et al. in that the rate of pleural thickening is lower in small effusions [14]. On the other hand, we found no relationship between the development of RPT and either tuberculin test results or microbiological findings in pleural fluid. Our results are in contrast with the study of Barbas et al. who considered that delayed type hypersensitivity is implicated in pleural fibrosis [9]. In addition, in our study there was no difference in LDH pleural fluid concentration, in accordance with the findings of Pablo et al., and in contrast with the findings of Soler et al. LDH is considered to be a reliable indicator of pleural inflammation. Finally, we found no differences in pleural fluid cell count, glucose, and $\mathrm{pH}[10,13]$.

ADA is an enzyme that catalyses the conversion of adenosine to inosine. ADA is a predominant T-lymphocyte enzyme and its plasma activity is high in diseases in which cellular immunity is stimulated. Demonstration of an elevated pleural fluid ADA level is useful in establishing the diagnosis of tuberculous pleuritis $[15,16]$. Several authors have used various cut off levels for the pleural fluid ADA between 33 and $50 \mathrm{U} / \mathrm{L}$ for the diagnosis of pleural tuberculosis [17]. The higher the pleural fluid ADA level, the more likely the patient is to have tuberculous pleuritis. There are two molecular forms of ADA - ADA1 and ADA2. ADA1 is found in all cells, but has its greatest activity in lymphocytes and monocytes. ADA2 is found in monocytes, and the majority of ADA in tuberculous pleuritis is ADA2. Consequently, separation of ADA into its isoenzymes slightly increases the sensitivity and specificity for the diagnosis of tuberculous pleuritis [18]. Uskul et al. showed that higher levels of total ADA and ADA 2 were seen in the group with pleural thickening and suggested that an immunological mechanism may play a role in the development of pleural thickening [14]. Interestingly, our study showed no difference in ADA pleural fluid concentration between the patients with or without RPT $>2 \mathrm{~mm}$. Similarly, our study showed no difference in ADA pleural fluid levels between the patients with RPT $\geq 10 \mathrm{~mm}$ and those with RPT $<10 \mathrm{~mm}$.

Vascular endothelial growth factor is a multifunctional cytokine that increases the permeability of the vasculature and is an important angiogenic and lymphogenic factor [19]. The most important factors for increasing VEGF expression are tissue inflammation, hypoxia, and transforming growth factor- $\beta$ levels. Although increased VEGF levels have been demonstrated in patients with malignancies, there are several reports showing increased levels of VEGF in patients with infectious diseases, especially pulmonary tuberculosis [20, 21]. It has been suggested that VEGF plays an impor- 
tant role in the development of granulomas. There are also several studies which show high of levels of the vascular endothelial growth factor in tuberculous pleural fluids [22-24]. When considering these results, we compared the levels of VEGF in patients with and without RPT as well as in the two subgroups of patients with RPT. Our results showed no difference in the levels of VEGF between these groups.

Finally, another test that is useful in the diagnosis of tuberculous pleuritis is the levels of interferon-gamma in the pleural fluid. Interferon-gamma is produced by the CD4+ lymphocytes from patients with tuberculous pleuritis. IFN- $\gamma$ is known to activate macrophages so that they increase their bactericidal capacity against $M$. tuberculosis [25]. Using a cut-off level of $3.7 \mathrm{IU} / \mathrm{mL}$, interferongamma values are very efficient in differentiating tuberculous from non-tuberculous effusions, presenting a sensitivity of $99 \%$ and a precision of $98 \%$, yet being more expensive than ADA [26]. To our knowledge, there is only one study in the literature which evaluated the relationship between the concentrations of IFN- $\gamma$ levels and the development of residual pleural thickening [27]. Interestingly, in our study there was no statistical difference in IFN- $\gamma$ concentration in pleural fluid of patients with or without RPT $>2 \mathrm{~mm}$ but a statistically significant difference in IFN- $\gamma$ concentration in pleural fluid of patients with RPT $\geq 10 \mathrm{~mm}$ and those with RPT $<10 \mathrm{~mm}$. However, ROC analysis did not identify a single cut-off point that presents an optimal combination of sensitivity and specificity for the development of significant RPT, but IFN- $\gamma$ values $>63.6 \mathrm{IU} / \mathrm{ml}$ present $90.5 \%$ specificity for RPT $\geq 10 \mathrm{~mm}$. Probably, larger studies with more cases with tuberculous pleuritis are needed to determine whether IFN- $\gamma$ levels can be used as a predictive parameter for the development of residual pleural thickening in tuberculous pleurisy.

In conclusion, our results showed that 25 out of 31 patients with pleural tuberculosis had residual pleural thickening when their therapy was completed. The incidence in our sample was comparable to that of previous reports, but our data suggests that the clinical characteristics or their pleural fluid findings may not provide information regarding which patients will develop residual pleural thickening. The levels of IFN- $\gamma$ in pleural fluid may represent one possible exception, since they were higher in the group with RPT $\geq 10 \mathrm{~mm}$ compared to the group with RPT $<10 \mathrm{~mm}$. Pleural fluid IFN- $\gamma$ may deserve further investigation in order to build up preventive and therapeutic strategies against RPT and its clinical complications.

\section{References}

1. Kumar D, Watson JM, Charlett A, Nicholas S, Darbyshire JH. Tuberculosis in England and Wales in 1993: results of a national survey. Thorax 1997; 52: 1060-1067.

2. Akotgu S, Yorgancioglu A, Cirak K, Kose T, Dereli SM. Clinical spectrum of pulmonary and pleural tuber- culosis: a report of 5480 cases. Eur Respir J 1996; 9: 2031-2035.

3. Chan CHS, Arnold M, Chan CY, et al. Clinical and pathological features of tuberculous pleural effusion and its long-term consequences. Respiration 1991; 58: 171-75.

4. Valdes L, San Jose E, Alvarez D, et al. Diagnosis of tuberculous pleurisy using the biologic parameters adenosine deaminase, lysozyme, and interferon gamma. Chest 1993; 103: 458-65.

5. Grove CS, Lee YC. Vascular endothelial growth factor: the key mediator in pleural effusion formation. Curr Opin Pulm Med 2002; 8: 294-301.

6. Hamed EA, EI-Noweihi AM, Mohamed AZ, Mahmound A. Vasoactive mediators (VEGF and TNF-alpha) in patients with malignant and tuberculous pleural effusions. Respirology 2004; 9: 81-86.

7. Giusti G. Adenosine deaminase. In: Bergmeyer HU, ed. Methods of enzymate analysis. New York, NY: Academic Press, 1974; 1092-1099.

8. Light RW. Pleural disease. 3rd ed. Baltimore: Williams \& Wilkins, 1990.

9. Lee $\mathrm{CH}$, Wang WJ, Lan RS, et al. Corticosteroids in the treatment of tuberculous pleurisy: a double-blind, placebo-controlled, randomised study. Chest 1988; 94 : 1256-59.

10. Barbas CS, Cukier A, Varvalho CR, Barbas Filho TV, Light RW. The relationship between pleural fluid findings and the development of pleural thichening in patients with pleural tuberculosis. Chest 1991; 100: 1264-67.

11. Soler JJ, Gil Suay V, Cordero P, et al. Corticoids en la tuberculosis pleural i Reducen el engrosamiento pleural residual[abstract]? Arch Bronconeumol 1994; 30 (suppl 1): 43.

12. Wyser C, Walzi G, Smedema J, et al. Corticosteroids in the treatment of tuberculous pleurisy. A double-blind, placebo-controlled, randomized study. Chest 1996; 110: 333-8.

13. Pablo A, Villena V, Echave-Sustaena J, Enchuetra Argel Lopez. Are pleural fluid parameters related to the development of residual pleural thickening in tuberculosis? Chest 1997; 112: 1293-1297.

14. Uskul B, Turker H, Ulman C, Ertgrul M. The relation of pleural thickening in tuberculosis pleurisy with the activity of adenosine deaminase. Monaldi Arch Chest Dis 2005; 63: 101-107.

15. Gourgoulianis K.I. Diagnostic value of adenosine deaminase activity in tuberculous effusions. Eur Res $J$ 1990; 3: 1098.

16. Ocana I, Martinez-Vazquez JM, Segura RM, Fernandez-De-Senllaj, Capdevila JA. Adenosine deaminase in pleural fluids: test for diagnosis of tuberculous pleural effusion. Chest 1983; 84: 51-53.

17. Burgess LJ, Maritz FJ, Le Roux I, Taljaard JJ. Use of adenosine deaminase as a diagnostic tool for tuberculous pleurisy. Thorax 1995; 50: 672-674.

18. Perez- Rodriguez E, Castro DJ. The use of ADA and ADA isoenzymes in the diagnosis of tuberculous pleurisy. Curr Opin 2000; 6: 259-266.

19. Cheng D, Rodriguez RM, Perkett EA, Rogers J, Bienvenu G, Light RW. Vascular endothelial growth factor in pleural fluid. Chest 1999; 115: 760-765.

20. Papaioannou AI, Kostikas K, Kollia P, Gourgoulianis KI. Clinical implications for vascular endothelial growth factor in the lung: friend or foe? Respir Res 2006; 7: 128.

21. Alatas F, Alatas Oz, Metintas M, et al. Vascular endothelial growth factor levels in active tuberculosis. Chest 2004; 125: 2156-2159.

22. Kraft A, Weinkel K, Ochs A, Marth C, et al. Vascular endothelial growth factor in the sera and effusions of 
patients with malignant and non-malignant disease. Cancer 1999; 85: 178-187.

23. Sack U, Hoffmann M, Zhao XJ, et al. Vascular endothelial growth factor in pleural effusions of different origin. Eur Respir J 2005; 25: 600-604.

24. Kiropoulos TS, Kostikas K,Gourgoulianis KI. Vascular endothelial growth factor levels in pleural and serum of patients with tubeculus pleural effusions. Chest 2005; 128: 468-469.

25. Ribera E, Ocana I, Martinez-Vazquez JM, Rossell M,
Espanol T, et al. High level of interferon gamma in tuberculous pleural effusions. Chest 1988; 93: 308-311.

26. Vilena V, Lopez-Encuentra A, Echave-Sustaeta J, et al. Interferon-gamma in 338 immunocompromized and immunocompetent patients for diagnosing pleural tuberculosis. Eur Respir J 1996; 9: 2635-2639.

27. Kim YK, Lee SY, Kwon SS, Kim KH, Moon HS, et al. Gamma-interferon and soluble interleukin 2 receptor in tuberculous pleural effusion. Lung 2001; 179: 175184.

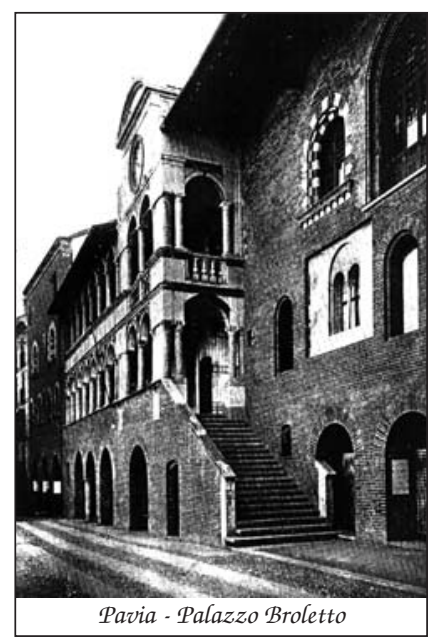

